\title{
$\underline{P-41}$
}

\section{Chemical Composition, Antioxidant and Antibacterial Activities of Syzygium Polyanthum (Wight) Walp. Essential Oils}

\author{
Nur Amalina A*, Natanamurugaraj G, Mashitah MY and Nurul Ashikin AK \\ Institution corresponding to authors: Faculty of Industrial Sciences and Technology, Universiti Malaysia Pahang, \\ 26300 Gambang, Kuantan, Pahang, Malaysia; E-mail: melamalina@gmail.com
}

This study was performed to determine the chemical composition, antioxidant and antibacterial activity of Syzygium polyanthum essential oil. The essential oil was extracted from the leaves by hydro distillation method. A total of 34 compounds were identified using GC-MS, representing $93.87 \%$ of the overall composition. The leaf essential oil of S. polyanthum was composed largely of the monoterpene hydrocarbons and sesquiterpene hydrocarbons. The main components of the oils were $\alpha-$ pinene $(30.88 \%)$ as major compounds followed by octanal $(18.30 \%)$ and $\alpha$-caryophyllene $(6.22 \%)$. Antioxidant activities of the essential oils were evaluated using three different assay; DPPH free radical scavenging, $\beta$-carotene bleaching and ferrous-ion chelating. Results showed that the oil exhibited a potential antioxidant activity. The antibacterial activity of the essential oil was tested using disc diffusion method against three Gram-positive and three Gram-negative bacterial strains and the minimum inhibitory concentration (MIC) was determined. The oil was found to be more active against Gram-negative bacteria than Gram-positive bacteria. This study concludes that the essential oil from $S$. polyanthum possess bioactivity potential for purpose of development of new natural-based products.

Keywords: Syzygium polyanthum, essential oil, GC-MS, antioxidant, antibacterial. 